\title{
In Silico Modeling of Ligand Molecule for Target Protein in Diabetes Mellitus Type II Insight Mechanism
}

\author{
Surya Prakash Dwivedi ${ }^{1}$, Neeraja Dwivedi ${ }^{1}$, R.B. $\operatorname{Singh}^{2}$, A. Srivastav ${ }^{3}$ and Sanjay Mishra, ${ }^{1, *}$ \\ ${ }^{1}$ Department of Biotechnology, College of Engineering \& Technology, IFTM Campus, Moradabad-244 001 (U.P.) India \\ ${ }^{2}$ Halberg Hospital and Research Institute, Civil Lines Moradabad 244001 (U.P.) India \\ ${ }^{3}$ Laboratory of Biomedical Engineering, College of Engineering \& Technology, IFTM Campus, Moradabad 244001 \\ (UP), India
}

\begin{abstract}
Type II Diabetes Mellitus or Non-Insulin dependent Diabetes mellitus (NIDDM) is one of the common diseases worldwide and often recognized as life style disease. The major symptom of this disease is an increase in blood glucose level resulting in polydypsia, polyuria and polyphagia. The cause of this abnormality is either lack of insulin production or insensitivity to the insulin produced inside the body. Glucagon like peptide (GLP) produced by the glucagons gene is responsible for insulin secretion. Thus proper functioning of GLP can be a remedy for Type II Diabetes mellitus. DiPeptidyl Peptidase IV (DPPIV) checks the GLP, thus in order to restore the normal functioning of these, the activity of DPP IV has to be checked using DPP IV inhibitors. In this paper the target sequence was retrieved, modeled using modeller $8 \mathrm{vl}$ followed by validation by PROCHECK. Ligand molecule was constructed by LIGBUILDER. Docking was carried out with methylamine as a lead molecule and DPPIV as receptor to design a drug molecule. Designing of a drug molecule was followed by screening for its activity and drug likeness. The present studies provide new insights for efficient inhibition of DPP IV to restore the normal activity of the body overcoming the negative effects left by other drugs.
\end{abstract}

Keywords: Diabetes, Glucagon like peptide, DiPeptidyl Peptidase IV, Drug, Ligbuilder, Molinspiration, Osiris.

\section{INTRODUCTION}

Diabetes mellitus type 2 (Non insulin-dependent diabetes (NIDDM), obesity related diabetes, or adult-onset diabetes) is a metabolic disorder that is primarily characterized by insulin resistance, relative insulin deficiency, and hyperglycemia [1]. It is often managed by engaging in exercise and modifying one's diet. Diabetes mellitus type 2 is often associated with obesity and hypertension and elevated cholesterol (combined hyperlipidemia), and with the condition Metabolic syndrome (also known as Syndrome X, Reavan's syndrome, or CHAOS). It is also associated with acromegaly, Cushing's syndrome and a number of other endocrinological disorders.

It is rapidly increasing in the developed world, and there is some evidence that this pattern will be followed in much of the rest of the world in coming years. The body requires energy in form of ATP to perform its regular metabolism efficiently this energy is stored in carbohydrates and glucose. When the energy is required, the stored energy is released via various pathways and chemical reactions. Thus, proper storage and usage of glucose is required in proper body functioning. When the glucose metabolism in the body is disturbed leading to an alteration in the glucose level, the

*Address correspondence to this author at the Department of Biotechnology, \& Dean (Research), College of Engineering \& Technology, IFTM Campus, Lodhipur Rajpoot, Delhi Road, Moradabad (U.P.) India;

Tel/Fax:+0091-591-2360818; E-mail: sanjaymishra66@gmail.com resulting disease is Diabetes. About 55 percent of type 2 are obese [2]. Chronic obesity leads to increased insulin resistance that can develop into diabetes, most likely because adipose tissue is a (recently identified) source of chemical signals (hormones and cytokines). Other research shows that type 2 diabetes causes obesity [3].

Glucagon like peptide is used for proper regulation of the insulin hormone for efficient usage and storage of the Glucose. Thus in order to efficiently control the glucose level in blood GLP expression has to be regulated.GLP-1 directly stimulates glucose-dependent insulin secretion via an increase in $\beta$-cell cAMP, through both protein kinase Adependent and independent mechanisms, with activation of signalling through small $\mathrm{G}$ proteins contributing to control of insulin exocytosis. GLP-1 receptor (GLP-1R) activation also promotes calcium mobilization and closure of the ATPsensitive KATP channel [4]. GLP 1 also stimulates beta cell proliferation. These observations raise the possibility that GLP-1 may be able to preserve or restore deteriorating $\beta$-cell function in type 2 diabetics in part via islet regeneration and augmentation of functional $\beta$-cell mass [5].

Dipeptidyl peptidase is used to check Glucagon like peptide over expression this helps to check the insulin secretion in turn . The principal known peptide substrates considered major targets of DPP-IV inhibitors when used for the treatment of diabetes are GLP-1 and GIP [6].

The inhibitor valine pyrrolidide (Val-pyr) reduced porcine plasma DPP-IV activity by $>90 \%$ and decreased the 
degradation of intact GLP-1, both in the fasted state and following exogenous GLP-1 administration. Infusion of glucose together with GLP-1 in the presence of Val-pyr produced a significant augmentation in levels of plasma insulin compared to GLP-1 infusion in the absence of the inhibitor.The DPP-IV inhibitor isoleucine thiazolidide (Ile-thiazolidide) prevented N-terminal degradation of both GLP-1 and GIP in human serum, and oral administration of Ile-thiazolidide to both lean and obese Zucker fatty rats inhibited plasma DPPIV activity, decreased glycaemic excursion and enhanced levels of circulating insulin following oral glucose loading. In contrast, administration of the inhibitor alone without concomitant glucose loading had no effect on levels of fasting glucose or insulin in obese Zucker rats [7]. More recent studies have examined the effects of chronic DPP-IV inhibitor administration in rodent models of type 2 diabetes. Oral administration of $\mathrm{P} 32 / 98,20 \mathrm{mg} / \mathrm{kg}$ b.i.d. for 3 months was associated with a progressive improvement in fasting glucose over the 12-week study period, in association with enhanced levels of glucose-stimulated insulin, a $12.5 \%$ decrease in relative body weight gain and improvements in insulin sensitivity as assessed at the end of the treatment period [8-12]. Pleiotropic functions of DPP-IV, acting as both a membrane bound and soluble form and exerting diverse effects on lymphocyte signaling, cell migration and proliferation, at times independent of its enzymatic activity, provide further challenges for scientists seeking to understand how specific inhibition of enzymatic function may impact the non-enzymatic biological actions of DPP-IV in different human tissues [13-15].

The conventional drug discovery approach is based on hit and trial methods and is often very time consuming, In addition to this; it may not lead to very efficient drugs necessarily. These shortcomings of this approach gave way to much faster and efficient method, the in silico drug designing. This approach makes use of the computed analysis of the target and various 3 dimensional possibilities of a particular structure. Not only does it make use of biology but also combines physics and chemistry involved for a drug formation. Fusion of various parameters leads to development of precise drugs and the process is precipitated with the use of computers. Also, the in silico process at the front end of lead discovery enables the pre-processing of compounds to detect their efficacy and characteristics. The approach used in this study is structure based making use of the structure of the ligand. Through this study we tried to design an inhibitor that would have least side effects and give maximum benefits overcoming the harms left by current day medicines as that of Metformin.

\section{METHODOLOGY}

\section{Comparative Protein Structure Modeling}

The sequence of the protein encoding for the disease (Diabetes Mellitus type 2) was retrieved from protein database of NCBI (http://www.ncbi.nlm.nih.gov/), (accession number CAA43118). The similarity search was performed by protein-protein blast with the Insight/ Homology module and adjusted to align key conserved residues [16]. The maximum similarity with $99 \%$ identity was observed with pdb2BGRA chain A, crystal structure of Hiv-1 tat derived Nonapeptides tat (1-9) bound to the active site of Dipeptidyl
Peptidase Iv (Cd26) 766 amino acid sequence. The 3D structure of the target protein was deduced employing the method of Threading and Fold Recognition method [17]. The modeling of 3D structure of protein encoding for the disease (Diabetes Mellitus type 2) following a stepwise procedure, was initiated with a template structure search from Protein databank (PDB) (http://www.rcsb.org/pdb/) related to the target sequence employing position-specific iterated BLAST (PSI-BLAST) [18]. From a number of hits, a potential template structure (PDB-ID: 1K30) representing the structure of Hiv-1 tat derived Nonapeptides tat was taken as template for model building.

For proceduring of fold assessment the sequence from the protein encoding for the disease (Diabetes Mellitus type 2) was submitted to the mGenThreader Server [19]. The server returned only one significant hit (PDB ID: 1K30) representing the structure of Hiv-1 tat derived Nonapeptides tat.

\section{Modeling of Protein Encoding for the Disease (Diabetes Mellitus Type 2)}

The template $(1 \mathrm{~K} 30)$ and target sequence were aligned using mGenThreader Server [19]. After careful examination for the potential alignment errors, the automated comparative protein modeling program MODELLER 9v2 (http://salilab. org/modeller) was employed to build the model. The statistical verification of the model was evaluated with PROCHECK.

\section{Active Sites Prediction and Probe Sphere Using PASS}

PASS was used for the prediction and visualization of the protein binding pockets [20]. The final protein structure selected after the analysis and validation step was used as an input in pdb format. Deep view was used to view these generated files to make the further process easier. ASPS file and PROBES file were used to find the amino acid resedue with minimum distance position from the active site.

\section{Virtual Screening of the Lead}

Lead molecule was selected for the generation of the drug. Methyl amine was used as the lead molecule. ChemSketch was used to design the drug molecule on computer to define its molecular structure.

\section{RIGID DOCKING}

Hex 4.5 was used for the purpose of docking of the lead with the target molecule [21]. The protein and the ligand were opened in the HEX and ligand was attached to the residue on the minimum distance position to the active site position. The docking controls were activated. The ligand was hence docked to the receptor protein. After docking was completed the position was saved in PDB format for the sub sequent steps.

\section{Generation and Optimization of Ligand}

Ligbuilder was used for generation of the ligands. It builds up ligand step by step using a library of organic fragments. The docked complex was provided as an input to the ligbuilder for the first step i.e. pocket generation [22]. This generated three important results: the pocket, the grid and the pharmacophore. The results from the pocket 
generation i.e. pocket and the grid and the Sybyl mol2 format of the ligand were given as an input. The populations and the ligands file in lig format was generated as the result. The result contained 5 ligands that were suitable for the conditions of a good ligand. Auto dock was used to predict the binding pattern of small molecules to the receptor of known 3D structure. It generally consists of 3 separate programs: auto dock performs the docking of the ligand to a set of grids describing the target protein; Auto grid is used for the pre calculation of these grids, and Auto tors sets up the bond that have to be treated as rotatable bonds in the ligand.

\section{RESULTS AND DISCUSSION}

\section{Target Sequence Retrieval}

The sequence of the protein encoding for dipeptidyl peptidase iv [Homo sapiens] with accession number CAA43118 was retrieved from NCBI. The similarity search was performed by protein-protein blast. The maximum similarity with $99 \%$ identity was observed with pdb2BGRA Chain A, Crystal Structure Of Hiv-1 Tat Derived Nonapeptides Tat (19) Bound To The Active Site Of Dipeptidyl Peptidase Iv (Cd26). The comparative $3 \mathrm{~d}$ modeling of protein structure was performed by modeller $8 \mathrm{vl}$. The modeller generated 5 models and the best model was validated and statistically analyzed by structure analysis and validation server (SAVS) [23]. The structures submitted were validated and the structure with core value of $90.6 \%$ and Zero bad contacts was used for the further process at lead target prediction. The model generated was visualized by Deep view and SPDv, which gave the idea of the 3 dimensional configuration of the protein, generated (Figs. 1A-1C).

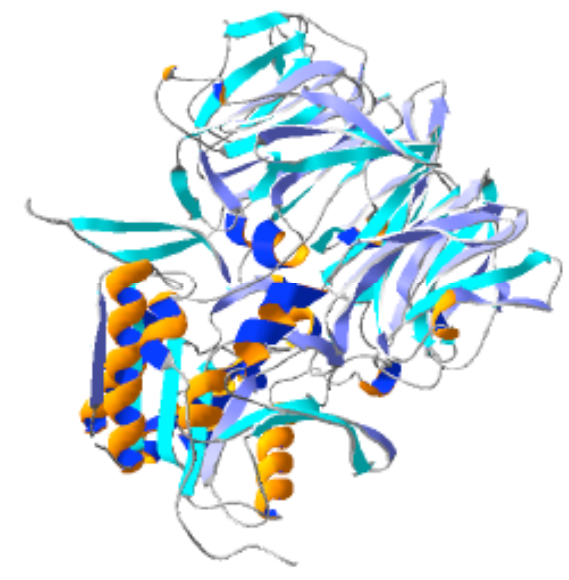

Fig. (1A). Second predicted model generated by modeller (ribbon structure).

The final protein structure selected after the analysis and validation step was used as input in PDB format. Deep View was used to view the regenerated files.

The protein and ligand were open in HEX and ligand was attached to the residue on the minimum distance position to the active site position (Figs. 2A, 2B). The ligand was hence docked to the receptor protein.

The ligbuilder tool was used for the inhibitor generation (Fig. 3). The result contained 5 ligands and a good ligand with suitable conditions was chosen.
The binding pattern, analyzed by AUTODOCK is used to predict of small molecule to the receptors of known 3D structure. The ligand and target protein were given as input and the docking was performed (Fig. 4).

After designing the drug molecule it was then screened for its activity and its drug likeness. Web based tools like MOLINSPIRATION and OSIRIS property explorer were used for this purpose. MOLINSPIRATION uses sophisticated Bayesian statistics to compare structures of representative ligands active on the particular target with structures of inactive molecules and to identify substructure features (which in turn determine physicochemical properties) typical for active molecules. Osiris draw chemical structures and calculates on-the-fly various drug-relevant properties whenever a structure is valid Prediction results are valued and color coded. Properties with high risks of undesired effects like mutagenicity or a poor intestinal absorption are shown in red. Whereas a green color indicates drug-conform behavior.

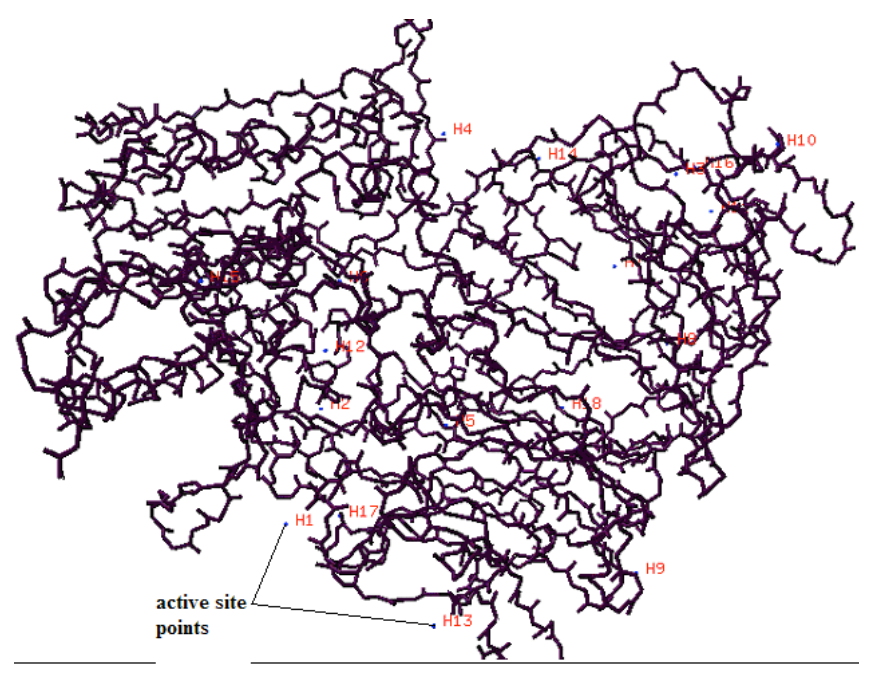

Fig. (1B). The Active site point (blue) where the ligand molecules can bind and develop into an efficient drug.

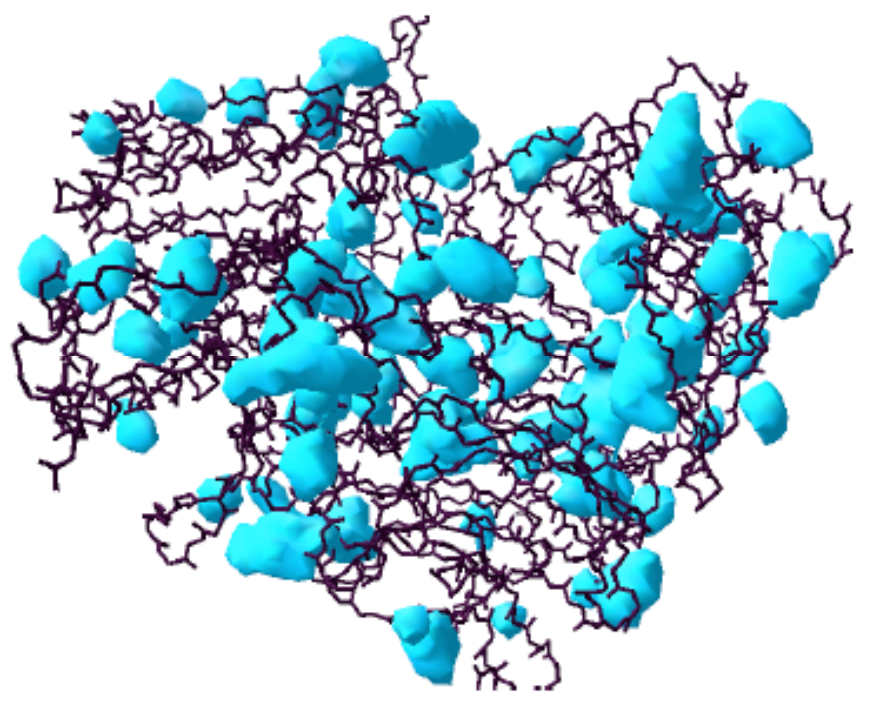

Fig. (1C). The 3D cavities around Probes (blue) where ligand binds. 


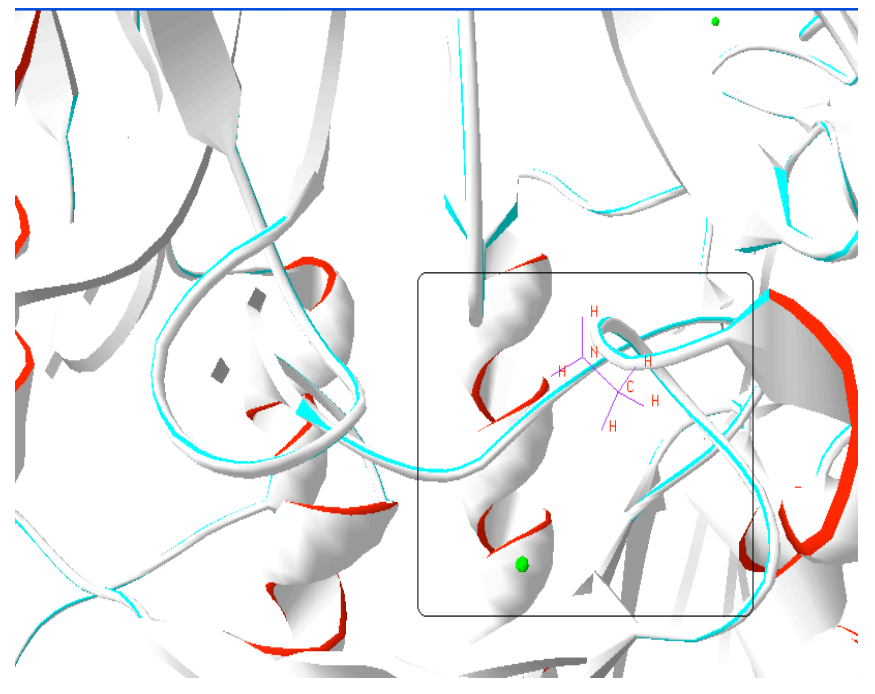

Fig. (2A). The ligand molecule (violet) inside the cavity.

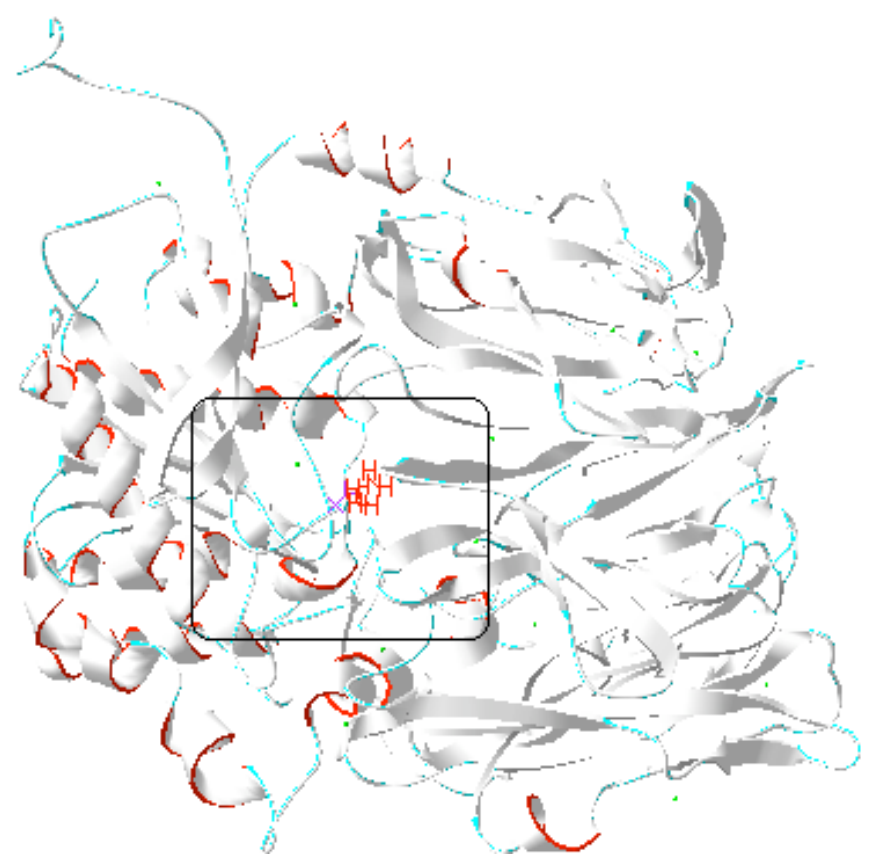

Fig. (2B). HEX result showing arrangement of seed molecule (shown with hydrogens- red) towards probe.

\section{CONCLUSION}

Diabetes now occupying a major segment of common man's ailments has traveled a long way from the first incidence of its occurrence. However, a permanent solution to the problems generated by it has not yet been reported. The major challenge on the way is the pleiotropic nature of DPPIV which can be a negative factor in its efficient incorporation as a drug. However, resolving this problem, there are not much hurdles to overcome in the process of Diabetes control. Thus with many problems still in hand to resolve, DPP IV inhibition serve as a milestone in controlling and eradication of TYPE 2 Diabetes mellitus. Moreover, this type of drug does not appear to have any side effect in the long run. Drugs as that of Metformin (biguanide) and sulphonyl ureas provided with some control of the disease however, the negative effects left behind by these drugs give them a weaker stand. DPP IV inhibition is an entirely different kind of approach trying to normalize the function of the body's natural glucose level controllers; hence they are more promising with lesser side effects and reactions.

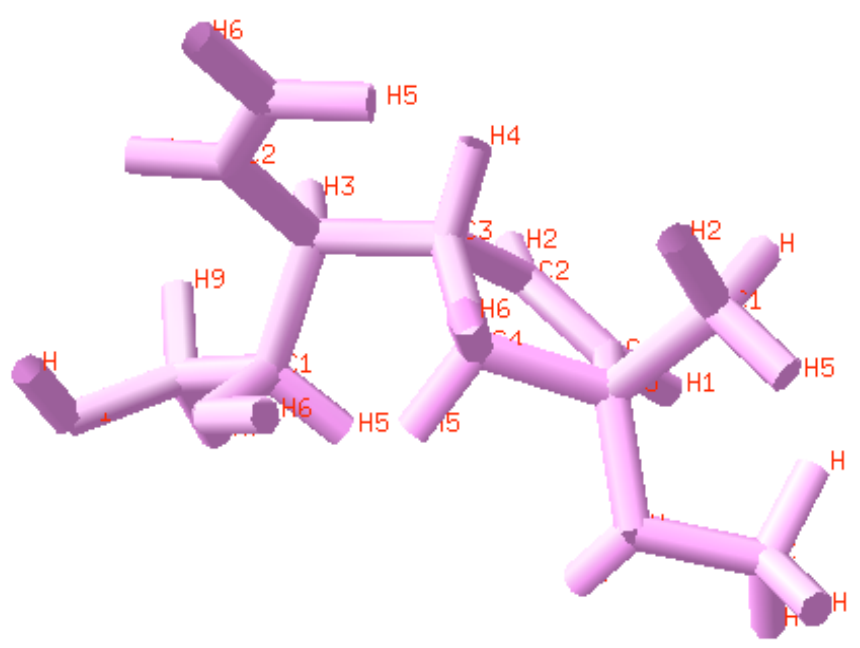

Fig. (3). Ligand molecule 2 developed by Ligbuilder.

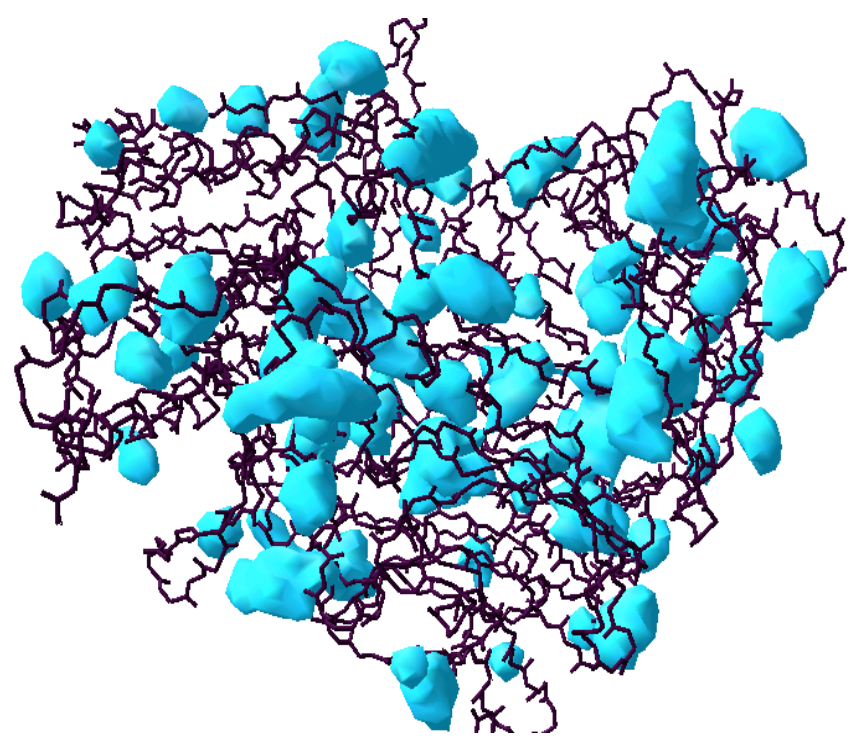

Fig. (4). Drug molecule along with all probes.

In order to make a contribution we tried to design a drug that would have fewer side effects compared to that with the currently available ones. Using In silico drug designing, the drug created has immense potential based on In silico parameters. Using structure based drug approach, the drug designed in this paper was developed using methylamine as a lead molecule. The results obtained by employing OSIRIS property explorer and MOLINSPIRATION project the generated molecule as a potential drug molecule with a drug score of 0.83 . The drug (3-[3-(methyl amino) cyclopentyl] pentane-1,2-diol) is observed to be non-mutagenic, non- irritant, non carcinogenic, non reproductive and a low molecular weight drug which means that may lead to an easy mobilization and rather higher bioavailability. 


\section{ACKNOWLEDGEMENT}

An institutional research promotion grant to the Department of Biotechnology, College of Engineering and Technology, Moradabad, U.P., India is acknowledged. The authors are grateful to Prof. R. M. Dubey (Managing Director, CET, IFTM, Moradabad, U.P, India) for providing the necessary facilities and encouragement. The authors are also thankful to Prof. B.N. Basu (Dean, Academics) and Prof. B.N. Kaul (Dean, Administration), College of Engineering and Technology, Moradabad, U.P., India, for their generous help and suggestions during the course of experimental work.

\section{REFERENCES}

[1] Himsworth H. Diabetes mellitus: its differentiation into insulinsensitive and insulin-insensitive types. Lancet 1936; 1: 127-30.

[2] Eberhart MS, Ogden C, Engelgau M. Prevalence of Overweight and Obesity Among Adults with Diagnosed Diabetes---United States, 1988-1994 and 1999-2002. MMWR Morb Mortal Wkly Rep 2004; 53(45): 1066-8.

[3] Camastra S, Borora E, Del Prato S. Effect of Obesity \& insulin resistance on resting and glucose induced thermogenesis in man. Int J Obes Relat Metab Disord 1999; 23(12): 1307-13.

[4] Effect of intensive blood-glucose control with metformin on complications in overweight patients with type 2 diabetes (UKPDS 34). UK Prospective Diabetes Study (UKPDS) Group Lancet 1998; 352(9131): 854-65.

[5] American Diabetes Association. Nutrition Recommendations and Interventions for Diabetes-2006. Diabetes Care 2006; 29: 2140-57.

[6] World Health Organisation Department of Noncommunicable Disease Surveillance. Definition, Diagnosis and Classification of Diabetes Mellitus and its Complications. 1999. Available from: whqlibdoc.who.int/hq/1999/WHO_NCD_NCS_99.2.pdf

[7] Ahrén B, Simonsson E, Larsson $\bar{H}$. Inhibition of dipeptidyl peptidase IV improves metabolic control over a 4-week study period in type 2 diabetes. Diabetes Care 2002; 25(5): 869-75.

[8] Pospisilik JA, Stafford SG, Demuth HU, et al. Long term treatment with the dipeptidyl peptidase IV inhibitor P32/ 98 causes sustained improvements in glucose tolerance, insulin sensitivity, hyperinsulinemia, and beta cell-glucose resposiveness in VDF (fa/fa) Zucher rates. Diabetes 2002; 21: 943-50.

[9] Department of Health (Malta), 1897-1972: Annual Reports. Available from: www.sahha.gov.mt/showdoc. aspx?id=589\& filesource $=4 \&$ file

[10] Diabetes Mellitus Classification \& Pathogenesis. Diabetes Mellitus \& Hypoglycemia. Armenian Medical Network (2006). Available from: www.diabetesdaily.com/wiki/Diabetes_mellitus

[11] Dobson M. Nature of the urine in diabetes. Med Observ Inquiries 1976; 5: 298-310.

[12] Drucker DJ. Therapeutic potential of dipeptidyl peptidase IV inhibitors for the treatment of type 2 diabetes. Expert Opin Investig Drugs 2003; 12(1) 93-6.

[13] Rother KI. Diabetes treatment, bridging the divide. N Engl J Med 2007; 356(15): 1499-501.

[14] Knowler W, Barrett-Connor E, Fowler S. Reduction in the incidence of type 2 diabetes with lifestyle intervention or metformin. N Engl J Med 2002; 346(6): 393-403.

[15] Stuebe AM, Rich-Edwards JW, Willett WC, Manson JE, Michels KB. Duration of lactation and incidence of type 2 diabetes. JAMA 2005; 294(20): 2601-10.

[16] Aasland R, Stewart AF, Gibson T. The SANT domain: a putative DNA-binding domain in the SWI-SNF and ADA complexes, the transcriptional co-repressor N-CoR and TFIIIB. Trends Biochem Sci 1996; 21: 87-88.

[17] Amores A, Force A, Yan YL, et al. Zebrafish hox clusters and vertebrate genome evolution. Science 1998; 282: 1711-4.

[18] Altschul SF, Madden TL, Schäffer AA, et al. Gapped BLAST and PSI-BLAST: a new generation of protein database search programs. Nucleic Acids Res 1997; 25: 3389-402.

[19] Bakshi R, Prakash T, Dash D, Brahmachari V. In silico characterization of the INO80 subfamily of SWI2/SNF2 chromatin remodeling proteins. Biochem Biophys Res Commun 2004; 320: 197-204.

[20] Aravind L, Iyer LM. The SWIRM domain: a conserved module found in chromosomal proteins points to novel chromatinmodifying activities. Genome Biol 2002; $3: 8$.

[21] Finn RD, Mistry J, Tate J, et al. The Pfam protein families database. Nucleic Acids Res 2004; 32: D138-41.

[22] Laskowski RA, MacArthur MW, Moss DS, Thornton JM. PROCHECK: a program to check the stereochemical quality of protein structures. J Appl Crystallogr 1993; 26: 283-91.

[23] Morris AL, MacArthur MW, Hutchinson EG, Thornton JM . Stereochemical quality of protein structure coordinates. Proteins 1992; 12: $345-64$. 